\title{
Giardia Duodenalis e Desnutrição Crónica em Crianças Menores de Cinco Anos de uma Região Rural da Guiné-Bissau
}

\author{
Giardia Duodenalis and Chronic Malnutrition in Children under Five \\ from a Rural Area of Guinea-Bissau
}

Sónia CENTENO-LIMA ${ }^{1,3}$, Vítor ROSADO-MARQUES ${ }^{2,9}$, Filipa FERREIRA ${ }^{1,3}$, Ruben RODRIGUES Renjamim $^{1,3}$, Benjam INDEQUE $^{4}$, Idrissa CAMARÁ ${ }^{4}$, Bruno de SOUSA ${ }^{5}$, Pedro AGUIAR ${ }^{6}$, Baltazar NUNES ${ }^{7}$, Paulo FERRINHO $^{8}$

Acta Med Port 2013 Nov-Dec;26(6):721-724

\begin{abstract}
RESUMO
Introdução: A desnutrição e as infecções por parasitas intestinais como Giardia duodenalis coexistem nas mesmas regiões geográficas, atingindo maiores prevalências em países em desenvolvimento. O ciclo da desnutrição e infecção implica que ambas as condições se podem agravar mutuamente e comprometer o crescimento e desenvolvimento das crianças, com especial relevância para as menores de cinco anos. O objectivo do presente estudo foi investigar a associação entre a desnutrição crónica e a infecção por G. duodenalis em crianças de uma comunidade rural da Guiné-Bissau.

Material e Métodos: Realizou-se um estudo de caso-controlo que incluiu 109 crianças com idades entre os 0 e os 59 meses de uma comunidade rural da Guiné-Bissau. A avaliação antropométrica das crianças em estudo permitiu identificar 31 casos de desnutrição crónica (z-score estatura para a idade <-2) e 78 controlos (z-score estatura para a idade $\geq-2$ ). Foi realizado exame microscópico de fezes para a detecção e identificação de $G$. duodenalis e de outros parasitas.

Resultados: Foi detectada infecção por G. duodenalis em 29,0\% (9/31) dos casos e 35,9\% (28/78) dos controlos. Não foi encontrada associação entre a infecção por $G$. duodenalis e a desnutrição crónica nas crianças em estudo.

Discussão e Conclusão: Os resultados obtidos mantêm em aberto o interesse em desenhar estudos que explorem esta associação em diferentes regiões e contextos epidemiológicos, apontando para a reflexão sobre a importância dos critérios de definição de desnutrição e concomitantemente da análise subsequente.
\end{abstract}

Palavras-chave: Criança; Giardia lamblia; Giardíase; Desnutrição; Guiné-Bissau.

\section{ABSTRACT}

Introduction: Malnutrition and infections by intestinal parasites such as Giardia duodenalis coexist in the same geographical regions, reaching the highest prevalence in developing countries. The cycle of malnutrition and infection implies that both conditions can aggravate each other and compromise the growth and development of children with special relevance for under-five. The aim of this study was to investigate the association between chronic malnutrition and infection by G. duodenalis in children under five in a rural community in Guinea-Bissau.

Material and Methods: A case-control study that included 109 children aged 0 to 59 months of a rural community in Guinea-Bissau was conducted. The anthropometric assessment of children in the study identified 31 cases of chronic malnutrition ( $z$-score height for age $<-2$ ) and 78 controls ( $z$-score height for age $\geq-2$ ). Microscopic examination of stools was performed for detection and identification of $G$. duodenalis and other parasites.

Results: The microscopic analysis of stool samples revealed G. duodenalis infection in $29.0 \%$ (9/31) of cases and $35.9 \%$ (28/78) of controls. No association between the infection with $\mathrm{G}$. duodenalis and chronic malnutrition in children under study could be established. Discussion and Conclusion: The results reinforce the interest in designing further studies exploring this association in different regions and epidemiological settings, while direct to the importance of the criteria for malnutrition definition which influences the subsequent analysis.

Keywords: Child; Giardia lamblia; Giardiasis; Malnutrition; Guinea-Bissau.

\section{INTRODUÇÃO}

A desnutrição crónica ou nanismo nutricional, identificada através do indicador comprimento ou estatura para a idade, inicia-se ainda no período de gestação devido à subnutrição materna, e continua durante a infância sendo causada por uma nutrição inadequada bem como por infecções frequentes que no seu conjunto não permitem à criança alcançar o potencial de crescimento linear. ${ }^{1,2}$
As infecções por parasitas intestinais, nomeadamente Giardia duodenalis, e a desnutrição crónica coexistem nas mesmas zonas geográficas. Este protozoário tem distribuição mundial, sendo um dos parasitas mais frequentes a infectar o Homem, com taxas de infecção geralmente baixas nos países desenvolvidos e atingindo $30 \%$ ou mais nos países em desenvolvimento. ${ }^{3} \mathrm{~A}$ infecção por G. duodena-

1. Unidade de Clínica Tropical. Instituto de Higiene e Medicina Tropical. Universidade Nova de Lisboa. Lisboa. Portugal.

2. Instituto de Investigação Científica Tropical. Lisboa. Portugal.

3. Centro de Malária e Doenças Tropicais. Instituto de Higiene e Medicina Tropical. Universidade Nova de Lisboa. Lisboa. Portugal.

4. Instituto da Biodiversidade e das Áreas Protegidas. Guiné. Guiné-Bissau.

5. Faculdade de Psicologia e de Ciências da Educação. Universidade de Coimbra. Coimbra. Portugal.

6. Departamento de Epidemiologia e Estatística. Escola Nacional de Saúde Pública. Universidade Nova de Lisboa. Lisboa. Portugal.

7. Departamento de Epidemiologia. Instituto Nacional de Saúde Dr. Ricardo Jorge. Lisboa. Portugal.

8. Unidade de Saúde Pública Internacional e Bioestatística. Instituto de Higiene e Medicina Tropical. Universidade Nova de Lisboa. Lisboa. Portugal.

9. Centro de Investigação em Antropologia e Saúde. Universidade de Coimbra. Coimbra. Portugal.

Recebido: 14 de Março de 2013 - Aceite: 19 de Julho de 2013 | Copyright $\odot$ Ordem dos Médicos 2013 
lis pode ser assintomática ou cursar com diarreia aguda ou crónica, dor abdominal, náusea e perda de peso, tendo sido reportadas elevadas taxas de reinfecção nas zonas endémicas. ${ }^{4,5}$ Considerando as consequências da infecção procurou-se explorar neste estudo de caso-controlo a exposição à infecção por $G$. duodenalis como factor de risco para a desnutrição crónica em crianças de uma zona rural da Guiné-Bissau.

\section{MATERIAIS E MÉTODOS}

Realizou-se um estudo de caso-controlo. A amostra foi composta por crianças com idades entre os 0-59 meses de 21 tabancas do Parque Natural das Lagoas de Cufada, Região Administrativa de Quinara da Guiné-Bissau. O estudo decorreu nos meses de Março e Abril de 2010, fim da época seca, no âmbito de um projecto de avaliação do estado nutricional e desenvolvimento infantil em crianças residentes no Parque e sua relação com os parasitas intestinais. Das 896 crianças das quais se fez a avaliação antropométrica, 249 tinham idades entre os 0 e os 59 meses, tendo sido seleccionados 31 casos (z-score estatura para a idade, EIZ<-2) e 78 controlos (EIZ $\geq-2)$, nas crianças de que se obteve amostra de fezes. A decisão sobre o número de crianças menores de cinco anos às quais seria realizada análise parasitológica de fezes prendeu-se exclusivamente com questões logísticas no terreno, decididas na planificação do estudo. Optou-se por estudar apenas uma criança por família, de modo a incorporar um maior número de agregados familiares, uma vez que é provável que exista uma maior variabilidade entre o estado nutricional das crianças de diferentes agregados familiares do que nas crianças pertencentes à mesma família. A selecção das crianças que entregaram as fezes foi feita pelos pais (normalmente a mãe) de acordo com a indicação de que teria de ser um rapaz ou uma rapariga, consoante a planificação, de modo a assegurar um equilíbrio entre os sexos.

O comprimento foi medido em decúbito dorsal nas crianças dos 0 aos 24 meses utilizando um estadiómetro Seca Mobile Measuring Mat for Babies and Toddlers (Model 210), com uma precisão de $0,5 \mathrm{~cm}$. A estatura das crianças com idade entre os 25 e os 59 meses foi medida de pé utilizando um estadiómetro portátil Seca (Model 214), com uma precisão de $0,1 \mathrm{~cm}$. Os dados obtidos foram introduzidos no software Anthro da OMS (http://www.who.int/ childgrowth/software/), permitindo obter directamente os z-scores para o comprimento ou estatura para a idade (EIZ), sendo considerada desnutrição crónica quando o indicador foi inferior a -2 z-scores, ${ }^{6}$ seguindo o critério da Organização Mundial de Saúde. ${ }^{7}$

As amostras de fezes (uma por criança) foram colhidas para contentores estéreis fornecidos às mães ou cuidadoras após explicação prévia do procedimento de colheita. As amostras foram transportadas em mala térmica com gelo, onde permaneceram 1 a $2 \mathrm{~h}$ até chegar ao laboratório no terreno onde foram imediatamente conservadas. Para esse efeito, aproximadamente $500 \mathrm{mg}$ de cada amostra de fezes fresca foi colocada em criotubos com cerca de $1 \mathrm{ml}$ do líquido de preservação e transporte Protofix ${ }^{T M}$ (Alpha-Tec Systems) mantidos à temperatura ambiente entre cerca de 5 a 20 dias, até serem transportados para Portugal, onde foram conservados entre 4 a $8^{\circ} \mathrm{C}$. A análise parasitológica de fezes, conduzida com vista à detecção de ovos, quistos e parasitas, foi efectuada por exame microscópico directo ${ }^{8}$ por três microscopistas independentes e em triplicado, para controlo de qualidade. Não se utilizou nenhum método de concentração.

Foi criada uma base de dados no programa SPSS (versão 18), e utilizados métodos de estatística descritiva bem como calculados os odds ratio e respetivos intervalos de confiança a $95 \%$ para medir o risco de exposição à infecção por G. duodenalis relativamente à desnutrição crónica. Foi utilizado o teste do Qui-Quadrado na análise de associação entre variáveis categóricas. Seguidamente procedeu-se a análise multivariável de dados utilizando o Software STATA 11 (http://www.stata.com/) recorrendo a modelação logística, impondo a entrada de todas as variáveis em simultâneo: exposição à infecção por $G$. duodenalis e eventuais factores de influência como o sexo e a idade, corrigido para o efeito de desenho associado à variável tabanca. $O$ controlo do efeito de desenho ${ }^{9}$ atribuível ao conglomerado da família foi assegurado através da inclusão de apenas uma criança por família. O estudo foi apresentado para apreciação às autoridades políticas da Região Administrativa de Quinara, bem com às autoridades de saúde e de educação locais de Buba, tendo sido obtida a aprovação para a realização do mesmo. Foi igualmente submetido ao Conselho de Ética do Instituto de Higiene e Medicina Tropical para apreciação, tendo sido aprovado.

\section{RESULTADOS}

As características demográficas dos 31 casos $(E I Z<-2)$ e 78 controlos ( $E I Z \geq-2$ ), descritas na Tabela 1, revelam que a maioria dos casos era do sexo masculino, e com idade superior a 24 meses.

A análise parasitológica de fezes demonstrou haver mais crianças infectadas com G. duodenalis nos controlos $(35,9 \%)$ do que nos casos $(29,0 \%)$,o que não foi estatisticamente significativo (Tabela 2). Três das infecções com G. duodenalis nos controlos eram mistas, dois com ancilostomídeos e um com Strongyloides stercoralis. Adicionalmente foi ainda detectada uma infecção simples com $S$. stercoralis num controlo.

Da regressão logística (OR ajustado) verificou-se que a infecção por G. duodenalis, no presente estudo, não constituiu um factor de risco estatisticamente significativo para a desnutrição crónica (Tabela 2). O efeito de desenho detectado associado a variável tabanca foi de 1,3.

\section{DISCUSSÃO}

O presente estudo de caso controlo foi desenvolvido com o objectivo de contribuir com evidências sobre a associação entre a infecção por Giardia duodenalis e a desnutrição crónica em crianças dos 0 aos 59 meses de uma região rural da Guiné-Bissau, o Parque Natural das Lagoas 
Tabela 1 - Caracterização demográfica da amostra: Crianças nos casos, nos controlos e total por sexo e por classe etária - frequências absolutas $(n)$ e frequências relativas $(\%)$.

\begin{tabular}{|c|c|c|c|}
\hline & $\begin{array}{c}\text { Casos } \\
n(\%)\end{array}$ & $\begin{array}{c}\text { Controlos } \\
n(\%)\end{array}$ & $\begin{array}{l}\text { Total } \\
n(\%)\end{array}$ \\
\hline \multicolumn{4}{|l|}{ Sexo* } \\
\hline Masculino & $22(71,0)$ & $31(39,7)$ & $53(48,6)$ \\
\hline Feminino & $9(29,0)$ & $47(60,3)$ & $56(51,4)$ \\
\hline \multicolumn{4}{|c|}{ Classe etária (meses) ${ }^{\star *}$} \\
\hline $0-24$ & $11(35,5)$ & $50(64,1)$ & $61(56,0)$ \\
\hline $24-59$ & $20(64,5)$ & $28(35,9)$ & $48(44,0)$ \\
\hline Total & 31 & 78 & 109 \\
\hline
\end{tabular}

Tabela 2 - Infeção por Giardia duodenalis nos casos e nos controlos, frequências absolutas (n), frequências relativas (\%), odds ratio (OR) não ajustado e ajustado e intervalo de confiança (IC 95\%)

\begin{tabular}{|c|c|c|c|c|c|c|}
\hline & $\begin{array}{c}\text { Casos (31) } \\
n(\%)\end{array}$ & $\begin{array}{c}\text { Controlos (78) } \\
n(\%)\end{array}$ & $\begin{array}{c}\text { OR } \\
\text { não ajustado }\end{array}$ & IC $95 \%$ & $\begin{array}{c}\text { OR } \\
\text { ajustado* }\end{array}$ & IC $95 \%$ \\
\hline $\begin{array}{l}\text { Infeção por } \\
\text { G. duodenalis }\end{array}$ & $9(29,0)$ & $28(35,9)$ & 0,731 & $0,296-1,802$ & 0,463 & $0,132-1,610$ \\
\hline
\end{tabular}

para a idade e sexo por regressão logística corrigida para o efeito de desenho associado à variável tabanca.

\section{da Cufada.}

Os resultados obtidos indicam que na amostra em estudo, a exposição à infecção por G. duodenalis não parece constituir factor de risco para a desnutrição crónica. Apesar de ter havido mais controlos infectados com $G$. duodenalis esta diferença não foi estatisticamente significativa. Acresce ainda que, de entre os 78 controlos $(E I Z \geq-2)$, cerca de $34,6 \%$ (27) têm um z-score de estatura para a idade (EIZ) entre -2 e -1, considerado por alguns autores como desnutrição crónica ligeira, ${ }^{10,11}$ estando $55,6 \%$ (15/27) infectados com G. duodenalis contra 25,5\% (13/51) dos eutróficos $(E I Z \geq-1)$. Verificou-se, assim, que as crianças com desnutrição crónica ligeira foram as que apresentaram maior frequência de infecção pelo parasita. Pese embora a limitada dimensão da amostra em estudo, estes dados apontam para a importância do critério de definição dos casos (EIZ <-2 ou EIZ <-1), podendo este facto ser integrado em estudos futuros, uma vez que na literatura se encontram resultados contraditórios no que concerne à contribuição da infecção por $G$. duodenalis para a desnutrição. ${ }^{12-15}$

Neste estudo, optou-se por considerar apenas como critério de infecção por $G$. duodenalis a visualização de quistos e/ou trofozoítos, uma vez que, mesmo considerando que métodos alternativos possam ser mais sensíveis, ${ }^{16,17}$ a microscopia permite adicionalmente a detecção simultânea de outros parasitas intestinais, ${ }^{18}$ que por si poderiam contri- buir para a desnutrição. ${ }^{19}$ No que se refere especificamente a G. duodenalis, importaria saber se as infecções eram sintomáticas ou assintomáticas. ${ }^{4,16}$ Tal informação, bem como o registo de comorbilidades e de factores associados à desnutrição crónica, ${ }^{20}$ a incluir em modelos de análise mais complexos, não foi recolhida no presente estudo.

\section{CONCLUSÕES}

No presente estudo não foi encontrada associação entre a infecção por $G$. duodenalis e a desnutrição crónica. Contudo, não obstante as limitações descritas, os resultados obtidos reforçam o interesse dos estudos sobre a desnutrição e a infecção, nomeadamente por parasitas intestinais, com uma amostra mais alargada, idealmente num estudo multicêntrico, conduzido em diferentes regiões e contextos epidemiológicos.

\section{AGRADECIMENTOS}

A Laura Cravo do Instituto de Higiene e Medicina Tropical (IHMT) pelo apoio técnico no diagnóstico laboratorial e a Inês Fronteira e Jorge Atouguia do IHMT, pelas contribuições para a discussão.

Ao Instituto da Biodiversidade e das Áreas Protegidas (IBAP) e a outras entidades locais que nos deram apoio logístico na recolha dos dados. 


\section{CONFLITOS DE INTERESSE}

Os autores declaram a inexistência de conflitos de interesse.

\section{REFERÊNCIAS}

1. Dewey KG, Begum K. Long-term consequences of stunting in early life. Matern Child Nutr. 2011;7:s5-18.

2. Dewey KG, Mayers DR. Early child growth: how do nutrition and infection interact? Matern Child Nutr. 2011;7:s129-42.

3. Feng Y, Xiao L. Zoonotic Potential and Molecular Epidemiology of Giardia Species and Giardiasis. Clin Microbiol Rev. 2011;24:110-40.

4. Farthing MJ, Cevallos AM, Kelly P. Intestinal protozoa. In: Cook G, Zumla A, editors. Manson's Tropical Diseases. 22nd ed. Saunders Elsevier; 2009. p.1375-406.

5. Gilman RH, Marquis GS, Miranda E, Vestegui M, Martinez H. Rapid reinfection by Giardia lamblia after treatment in a hyperendemic Third World community. Lancet. 1988;1:343-5.

6. de Onis M, Onyango AW, Borghi E, Garza C, Yang H. World Health Organization Multicentre Growth Reference Study Group. Comparison of the WHO Child Growth Standards and the National Center for Health Statistics/WHO international growth reference: implications for child health programmes. Public Health Nutr. 2006;9:942-7.

7. World Health Organization. Global Database on Child Growth and Malnutrition [Consultado em 2013 Jul 17]. Disponível em: http://www.who. int/nutgrowthdb/about/introduction/en/index5.html.

8. World Health Organization. Bench aids for the diagnosis of intestinal parasites. Geneva: WHO; 1994.

9. Last J. A dictionary of epidemiology. 3rd ed. Oxford: Oxford University Press; 1995.

10. Oliveira Assis AM, Barreto ML, Magalhães de Oliveira LP, de Oliveira VA, da Silva Prado M, da Silva Gomes GS, et al. Determinants of mildto-moderate malnutrition in preschoolers in an urban area of Northeastern Brazil: a hierarchical approach. Public Health Nutr. 2008;11:387-94.

11. Stevens GA, Finucane MM, Paciorek CJ, Flaxman SR, White RA, Donner AJ, et al. Trends in mild, moderate, and severe stunting and underweight, and progress towards MDG 1 in 141 developing countries: a systematic analysis of population representative data. Lancet.

\section{FONTES DE FINANCIAMENTO}

Subsídios ou bolsas que contribuíram para a realização do trabalho: A Fundação Luso Americana para o Desenvolvimento apoiou financeiramente a deslocação à Guiné-Bissau de Vítor Rosado Marques.

2012;380:824-34.

12. Hollm-Delgado MG, Gilman RH, Bern C, Cabrera L, Sterling CR, Black $\mathrm{RE}$, et al. Lack of an adverse effect of Giardia intestinalis infection on the health of Peruvian children. Am J Epidemiol. 2008; 168:647-55.

13. Muniz-Junqueira MI, Queiroz EF. Relationship between protein-energy malnutrition, vitamin A, and parasitoses in living in Brasilia. Rev Soc Bras Med Trop. 2002;35:133-41.

14. Botero-Garces JH, Garcia-Montoya GM, Grisales-Patino D, Aguirre-Acevedo DC, Alvarez-Uribe MC. Giardia intestinalis and nutritional status in children participating in the complementary nutrition program, Antioquia, Colombia, May to October 2006. Rev Inst Med Trop Sao Paulo. 2009;51:155-62.

15. Sackey ME, Weigel MM, Armijos RX. Predictors and nutritional consequences of intestinal parasitic infections in rural Ecuadorian children. $J$ Trop Pediatr. 2003:49:17-23.

16. Ignatius R, Gahutu JB, Klotz C, Steininger C, Shyirambere C, Lyng M, et al. High prevalence of Giardia duodenalis Assemblage B infection and association with underweight in Rwandan children. PLoS Negl Trop Dis. 2012;6:e1677.

17. Ferreira FS, Centeno-Lima S, Gomes J, Rosa F, Rosado V, Parreira $\mathrm{R}$, et al. Molecular characterization of Giardia duodenalis in children from the Cufada Lagoon Natural Park, Guinea-Bissau. Parasitol Res. 2012;111:2173-7.

18. Schuurman $\mathrm{T}$, Lankamp $\mathrm{P}$, van Belkum $\mathrm{A}$, Kooistra-Smid $\mathrm{M}$, van Zwet $\mathrm{A}$ Comparison of microscopy, real-time PCR and a rapid immunoassay for the detection of Giardia lamblia in human stool specimens. Clin Microbiol Infect. 2007;13:1186-91.

19. Hall A, Hewitt G, Tuffrey V, de Silva N. A review and meta-analysis of the impact of intestinal worms on child growth and nutrition. Matern Child Nutr. 2008;4:s118-236.

20. Frongillo EA Jr. Symposium: Causes and etiology of stunting. introduction. J Nutr. 1999;129:S529-30. 
Sónia CENTENO-LIMA, Vítor ROSADO-MARQUES, Filipa FERREIRA, Ruben RODRIGUES, Benjamim INDEQUE, Idrissa CAMARÁ, Bruno de SOUSA, Pedro AGUIAR, Baltazar NUNES, Paulo FERRINHO

\section{Giardia Duodenalis e Desnutrição Crónica em Crianças Menores de Cinco Anos de uma Região Rural da Guiné-Bissau \\ Acta Med Port 2013:26:721-724}

Publicado pela Acta Médica Portuguesa, a Revista Científica da Ordem dos Médicos

Av. Almirante Gago Coutinho, 151

1749-084 Lisboa, Portugal.

Tel: +351218428 215

E-mail: submissao@actamedicaportuguesa.com

www.actamedicaportuguesa.com

ISSN:0870-399X | e-ISSN: 1646-0758

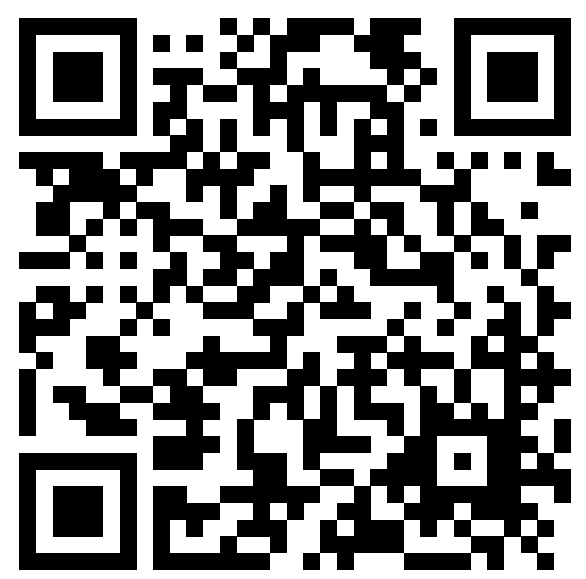

